\title{
Study of the correlation between growth hormone deficiency and serum leptin, adiponectin, and visfatin levels in adults
}

\author{
Z.-P. Li ${ }^{1}$, M. Zhang' ${ }^{2}$ J. Gao ${ }^{3}$, G.-Y. Zhou ${ }^{3}$, S.-Q. Li ${ }^{1}$ and Z.-M. An ${ }^{3}$ \\ ${ }^{1}$ Golden Card Hospital, West China Hospital, Sichuan University, \\ Chengdu, China \\ ${ }^{2}$ Department of Laboratory Medicine, West China Hospital, \\ Sichuan University, Chengdu, China \\ ${ }^{3}$ Department of Endocrinology, West China Hospital, \\ Sichuan University, Chengdu, China \\ Corresponding authors: S.-Q. Li / Z.-M. An \\ E-mail: lishuangqing201@yeah.net / anzhenmei2012@yeah.net
}

Genet. Mol. Res. 13 (2): 4050-4056 (2014)

Received July 10, 2013

Accepted November 13, 2013

Published February 14, 2014

DOI http://dx.doi.org/10.4238/2014.February.14.16

\begin{abstract}
We aimed to determine the significance and changes in leptin, adiponectin (ADP), and visfatin levels in adults with growth hormone deficiency (GHD). Forty adults (19 men, 21 women) who had been diagnosed with GHD comprised the observation group, while 36 healthy adults (18 men, 18 women) were used as the control group. Fasting venous blood was collected to detect leptin, ADP, and visfatin levels. There was no statistically significant difference ( $\mathrm{P}$ $>0.05)$ between the GHD group and the control group in terms of gender ratio, age, and body mass index. The waist-to-hip ratio (0.894 \pm 0.061 vs $0.830 \pm 0.481)$, cholesterol $(4.99 \pm 1.046$ vs $4.18 \pm 0.683)$, triglyceride $(1.97 \pm 1.428$ vs $1.08 \pm 0.403)$, LDL (2.91 \pm 0.980 vs 2.29 $\pm 0.540)$, leptin $(3.00 \pm 1.233$ vs $1.89 \pm 1.554)$, ADP $(15.26 \pm 6.449$ vs $10.24 \pm 7.608)$, and visfatin levels $(10.42 \pm 3.715$ vs $5.87 \pm 3.90)$ in the GHD group were significantly higher than those in the control
\end{abstract}


group (all $\mathrm{P}<0.05)$. The levels of growth hormone $(1.68 \pm 1.67 \mathrm{vs}$ $15.53 \pm 6.23$ ), insulin-like growth factor-1 (IGF-1, $22.64 \pm 16.41 v s$ $61.85 \pm 28.48)$, IGF-binding protein-3 $(4889 \pm 2962$ vs $6866 \pm 3823)$, and dehydroepiandrosterone sulfate $(1.466 \pm 1.804$ vs $6.000 \pm 2.767)$ in the GHD group were significantly lower than those in the control group (all $\mathrm{P}<0.05$ ). Correlation analysis demonstrated that leptin level was positively correlated to ADP and visfatin in both the GHD and control groups and negatively correlated to IGF-1 $(r=0.332, \mathrm{P}<0.05)$. Logistic regression analysis demonstrated that leptin, ADP, and visfatin were independent risk factors for adults with GHD.

Key words: Adult GHD; Leptin; Adiponectin; Visfatin

\section{INTRODUCTION}

Growth hormone deficiency (GHD) is an endocrine disease with multiple clinical features induced by a group of factors. Recent research has demonstrated that adult GHD not only affects physical growth and quality of life, but also significantly increases the rate of mortality, in particular, mortality induced by angiocardiopathy (Bulow et al., 2000).

It has been reported that in adult GHD, body fat content and body weight increase and muscle amount decreases significantly, accompanied by abnormal triglyceride levels, insulin resistance, and glucose tolerance (McCallum et al., 2002). Some researchers believed that the abnormal increase of body and blood fat was closely related to the secretion and metabolism of fat tissue (Alghasham and Barakat, 2008). Some studies have reported that as both an energy store and endocrine organ, fat tissue participates in the regulation of an organism's energy metabolism and inflammation by secreting multiple fat cell factors. It has been established that fat cell factors such as leptin, adiponectin (ADP), and visfatin influence body fat content and induce abnormal secretion functions of blood fat and insulin resistance.

How do the levels of multiple fat factors change in adult GHD? Are there alterations to the mechanisms of occurrence of body fat formation, leading to abnormal fat and blood fat levels relative to the altered levels of multiple fat factors? These issues have become the focus of research in recent years.

At present, the causes of adult GHD and the mechanisms of occurrence of its related clinical features have yet to be defined. Regarding the alteration of multiple fat factors in adult GHD and their correlation to growth hormone (GH), related research involving small samples has been carried out in other countries but with inconsistent conclusions; currently, there is no domestic research on the issue.

\section{MATERIAL AND METHODS}

\section{Subjects}

The GHD group was composed of adults diagnosed with GHD at our hospital by a clinical insulin tolerance test (ITT). There were 40 patients (19 men, 21 women). The inclusion criteria were 1) age 20-60 years, 2) diagnosis of GHD by clinical ITT, 3) no severe dis- 
eases such as hepatopathy, nephropathy, cardiopathy, or psychopathy, and 4) not pregnant or breastfeeding.

The control group included healthy adults undergoing physical examination at our physical examination center, and who had no abnormality according to chest film or type-B ultrasonic, electrocardiogram, and biochemical examinations. There were 36 adults (18 men, 18 women) in the control group, and all subjects, including GHD patients, provided informed consent. The inclusion criteria were 1) age 20-60 years, 2) healthy people undergoing physical examination at the hospital's physical examination center, 3) no severe disease history such as cancer, diabetes, hepatopathy, nephropathy, cardiopathy, or psychopathy, 4) not pregnant or breastfeeding.

\section{Data collection}

General data collected were gender, age, chief complaint, clinical features, life habits, and disease history. Physical measurements included height, weight, waistline, and hipline measured in a fasting state, and the data were used to determine the body mass index (BMI). BMI = weight $(\mathrm{kg}) /$ height $^{2}(\mathrm{~m})$; waist-to-hip ratio $(\mathrm{WHR})=$ waistline $(\mathrm{cm}) /$ hipline $(\mathrm{cm})$. All data were recorded for statistical analysis.

\section{Sampling}

Fasting venous blood was collected from all subjects for routine blood, liver function, kidney function, whole blood fat, fasting blood glucose, thyroid hormone, insulin, and gonadal hormone tests. The remainder of the samples was separated by centrifuging for $15 \mathrm{~min}$ at 1000 $\mathrm{rpm}$ and stored at $-70^{\circ} \mathrm{C}$ for leptin, $\mathrm{ADP}$, and visfatin detection.

\section{Leptin, ADP, and visfatin detection}

Kits for leptin, ADP, and visfatin detection were purchased from R\&D Systems (USA) and detection was carried out according to the kit instructions.

\section{Statistical analysis}

SPSS 13.0 (Chicago, IL, USA) was used for statistical analysis. Normal distribution data are reported as means \pm standard deviation, while skewed distribution data are reported as the median (interquartile range). Independent sample $t$-test and chi-square tests were used for comparison between the two groups. Pearson's correlation analysis was used for correlation analysis among indices. Logistic regression analysis was used for forecasting risk. $\mathrm{P}<0.05$ indicated statistical significance.

\section{RESULTS}

\section{Baseline characteristics of GHD and control groups}

Equilibrium comparison of gender between the GHD and control groups is listed in Table 
1. There was no significant difference in the gender ratios between the two groups $(\mathrm{P}>0.05)$. WHR, cholesterol (CHOL), triglyceride (TG), and LDL levels in the GHD group were higher than those in the control group $(\mathrm{P}<0.05)$; GH, insulin-like growth factor-1 (IGF-1), IGF-binding protein-3 (IGFBP-3), and dehydroepiandrosterone sulfate (DHEA-S) levels in the GHD group were lower than those in the control group $(\mathrm{P}<0.05)$. The levels of leptin, ADP, and visfatin in the GHD group were higher than those in the control group $(\mathrm{P}<0.05)$. There was no statistical significance between the GHD group and the control group for age, BMI, insulin, C-peptide, and HDL (Table 2).

\begin{tabular}{|c|c|c|c|c|c|}
\hline \multirow[t]{2}{*}{ Group } & \multicolumn{2}{|c|}{ Gender } & \multirow[t]{2}{*}{ Sum } & \multirow[t]{2}{*}{$\chi^{2}$ value } & \multirow[t]{2}{*}{$P$ value } \\
\hline & Male & Female & & & \\
\hline GHD group & 19 & 21 & 40 & 0.047 & 0.828 \\
\hline Control group & 18 & 18 & 36 & & \\
\hline Sum & 37 & 39 & 76 & & \\
\hline
\end{tabular}

Table 2. Baseline characteristics between growth hormone deficiency (GHD) and control groups.

\begin{tabular}{lccc}
\hline & GHD group $(\mathrm{N}=40)$ & Control group $(\mathrm{N}=36)$ & P value \\
\hline Age $($ years $)$ & $38.30 \pm 8.561$ & $37.19 \pm 10.899$ & 0.625 \\
BMI $\left(\mathrm{kg} / \mathrm{m}^{2}\right)$ & $22.39 \pm 4.209$ & $22.26 \pm 1.520$ & 0.851 \\
WHR & $0.894 \pm 0.061$ & $0.830 \pm 0.481$ & 0.000 \\
Cholesterol $(\mathrm{mM})$ & $4.99 \pm 1.046$ & $4.18 \pm 0.683$ & 0.000 \\
Glycerin trilaurate $(\mathrm{mM})$ & $1.97 \pm 1.428$ & $1.08 \pm 0.403$ & 0.000 \\
High-density lipoprotein $(\mathrm{mM})$ & $1.39 \pm 0.549$ & $1.45 \pm 0.310$ & 0.552 \\
Low-density lipoprotein $(\mathrm{mM})$ & $2.91 \pm 0.980$ & $2.29 \pm 0.540$ & 0.001 \\
IGFBP-3 $(\mu \mathrm{g} / \mathrm{L})$ & $4889 \pm 2962$ & $6866 \pm 3823$ & 0.015 \\
DHEA-S $(\mu \mathrm{M})$ & $1.466 \pm 1.804$ & $6.000 \pm 2.767$ & 0.000 \\
IGF-1 $(\mu \mathrm{g} / \mathrm{L})$ & $22.64 \pm 16.41$ & $61.85 \pm 28.48$ & 0.000 \\
GH $(\mu \mathrm{g} / \mathrm{L})$ & $1.68 \pm 1.67$ & $15.53 \pm 6.23$ & 0.000 \\
Fasting insulin $(\mu \mathrm{U} / \mathrm{L})$ & $4.80 \pm 2.66$ & $4.04 \pm 2.37$ & $0.61 \pm 0.09$ \\
Fasting C-peptide $(\mathrm{nM})$ & $0.57 \pm 0.09$ & $19.81 \pm 5.35$ & 0.065 \\
Insulin of 2 h after meal $(\mu \mathrm{U} / \mathrm{L})$ & $19.32 \pm 5.49$ & $1.74 \pm 0.35$ & 0.690 \\
C-peptide of 2 h after meal $(\mathrm{nM})$ & $1.72 \pm 0.35$ & $1.89 \pm 1.554$ & 0.818 \\
Leptin $(\mu \mathrm{g} / \mathrm{L})$ & $3.00 \pm 1.233$ & $10.24 \pm 7.608$ & 0.001 \\
ADP $(\mu \mathrm{g} / \mathrm{mL})$ & $15.26 \pm 6.449$ & $5.87 \pm 3.90$ & 0.003 \\
Visfatin $(\mu \mathrm{g} / \mathrm{L})$ & $10.42 \pm 3.715$ & 0.000 \\
\hline BMI $/ \mathrm{b}$ ) & & & \\
\hline
\end{tabular}

BMI = body mass index; WHR = waist-to-hip ratio; IGFBP-3 = insulin-like growth factor-binding protein-3; DHEA-S = dehydroepiandrosterone sulfate; IGF-1 = insulin-like growth factor-1; GH = growth hormone; ADP $=$ adiponectin.

\section{Correlation analysis of leptin, ADP, and visfatin with metabolic indices in the GHD group}

Leptin was correlated positively to ADP and visfatin and negatively to IGF-1. Leptin was not correlated to age, BMI, WHR, blood fat, body fat content, GH, IGFBP-3, postprandial C-peptide (PTC), thyroid-stimulating hormone (TSH), free triiodothyronine (FT3), free thyroxine (FT4), luteinizing hormone (LH), follicle-stimulating hormone (FSH), prolactin (PRL), estradiol (E2), testosterone (T), DHEA-S, insulin, or C-peptide.

ADP was correlated positively to leptin and visfatin but was not correlated to age, BMI, WHR, blood fat, body fat content, GH, IGF-1, IGFBP-3, PTC, TSH, FT3, FT4, LH, FSH, PRL, E2, T, DHEA-S, insulin, or C-peptide. 
Visfatin was correlated positively to ADP and leptin $(\mathrm{r}=0.830, \mathrm{P}<0.05)$ but negatively to IGF-1 $(\mathrm{r}=0.336, \mathrm{P}<0.05)$. Visfatin was not correlated to age, BMI, WHR, blood fat, body fat content, GH, IGF-1, IGFBP-3, PTC, TSH, FT3, FT4, LH, FSH, PRL, E2, T, DHEA$\mathrm{S}$, insulin, or C-peptide (Table 3).

\begin{tabular}{|c|c|c|c|c|c|c|}
\hline \multirow[t]{2}{*}{ GHD group $(\mathrm{N}=40)$} & \multicolumn{2}{|c|}{ Leptin } & \multicolumn{2}{|c|}{ ADP } & \multicolumn{2}{|c|}{ Visfatin } \\
\hline & $\mathrm{r}$ value & P value & $\mathrm{r}$ value & $\overline{P \text { value }}$ & $\mathrm{r}$ value & $P$ value \\
\hline Age & 0.082 & 0.616 & 0.056 & 0.732 & 0.025 & 0.877 \\
\hline BMI & 0.082 & 0.616 & 0.111 & 0.495 & 0.058 & 0.722 \\
\hline WHR & -0.101 & 0.536 & -0.133 & 0.414 & 0.030 & 0.857 \\
\hline Cholesterol & 0.045 & 0.781 & -0.056 & 0.730 & 0.022 & 0.892 \\
\hline Glycerin trilaurate & -0.036 & 0.823 & -0.113 & 0.489 & 0.057 & 0.726 \\
\hline High-density lipoprotein & 0.154 & 0.344 & 0.095 & 0.559 & -0.009 & 0.958 \\
\hline Low-density lipoprotein & 0.076 & 0.642 & 0.026 & 0.872 & 0107 & 0.512 \\
\hline Body fat content & 0.025 & 0.877 & -0.095 & 0.558 & -0.023 & 0.888 \\
\hline Cortisol & -0.129 & 0.441 & 0.001 & 0.995 & -0.076 & 0.652 \\
\hline Growth hormone & 0.110 & 0.500 & 0.025 & 0.875 & 0.143 & 0.379 \\
\hline Fasting insulin & -0.104 & 0.525 & -0.088 & 0.591 & -0.182 & 0.260 \\
\hline Fasting C-peptide & 0.190 & 0.240 & 0.160 & 0.323 & 0.153 & 0.347 \\
\hline Insulin of $2 \mathrm{~h}$ after meal & -0.021 & 0.898 & 0.078 & 0.631 & -0.040 & 0.806 \\
\hline C-ppeptide of $2 \mathrm{~h}$ after meal & -0.014 & 0.933 & -0.012 & 0.941 & 0.121 & 0.456 \\
\hline IGF-1 & -0.332 & 0.036 & -0.296 & 0.064 & -0.335 & 0.035 \\
\hline IGFBP-3 & 0.042 & 0.795 & 0.082 & 0.614 & 0.245 & 0.128 \\
\hline TSH & -0.090 & 0.603 & -0.056 & 0.745 & -0.022 & 0.896 \\
\hline FT3 & 0.128 & 0.456 & 0.159 & 0.354 & 0.171 & 0.319 \\
\hline FT4 & 0.078 & 0.651 & 0.101 & 0.559 & 0.048 & 0.783 \\
\hline Luteinizing hormone & -0107 & 0.540 & -0.028 & 0.872 & -0.191 & 0.272 \\
\hline Follicule-stimulating hormone & -0.091 & 0.598 & -0.038 & 0.824 & -0.202 & 0.237 \\
\hline Prolactin & 0.254 & 0.134 & 0.260 & 0.125 & 0.195 & 0.255 \\
\hline Estradiol & -0.074 & 0.671 & -0.121 & 0.490 & 0.032 & 0.857 \\
\hline Testosterone & -0.215 & 0.238 & -0.099 & 0.591 & -0.078 & 0.671 \\
\hline DHEA-S & -0.094 & 0.563 & -0.105 & 0.520 & -0.006 & 0.973 \\
\hline Leptin & - & - & 0.895 & 0.000 & 0.830 & 0.000 \\
\hline $\mathrm{ADP}$ & 0.895 & 0.000 & - & - & 0.769 & 0.000 \\
\hline Visfatin & 0.830 & 0.000 & 0.769 & 0.000 & - & - \\
\hline
\end{tabular}

BMI = body mass index; WHR = waist-to-hip ratio; IGF-1 = insulin-like growth factor-1; IGFBP-3 = insulin-like growth factor-binding protein-3; TSH = thyroid stimulating hormone; FT3 = free triiodothyronine; FT4 = free thyroxine; DHEA-S = dehydroepiandrosterone sulfate.

\section{Correlation analysis of leptin, ADP, and visfatin with metabolic indices in the control group}

Leptin was correlated positively to TG, ADP, and visfatin but negatively to IGFBP-3. Leptin was not correlated to age, BMI, WHR, CHOL, HDL, LDL, GH, IGF-1, DHEA-S, insulin, or C-peptide.

ADP was positively correlated to TG, leptin, and visfatin but negatively to IGFBP-3. ADP was not correlated to age, BMI, WHR, CHOL, HDL, LDL, GH, IGF-1, DHEA-S, insulin, or C-peptide.

Visfatin was correlated positively to TG, ADP, and leptin but negatively to IGFBP-3 and 2-h postprandial insulin level. Visfatin was not correlated to age, BMI, WHR, CHOL, HDL, LDL, GH, IGF-1, DHEA-S, insulin, or C-peptide (Table 4). 


\begin{tabular}{|c|c|c|c|c|c|c|}
\hline \multirow[t]{2}{*}{ Control group $(\mathrm{N}=36)$} & \multicolumn{2}{|c|}{ Leptin } & \multicolumn{2}{|c|}{$\mathrm{ADP}$} & \multicolumn{2}{|c|}{ Visfatin } \\
\hline & r value & P value & $\mathrm{r}$ value & $P$ value & $\mathrm{r}$ value & $P$ value \\
\hline Age & -0.031 & 0.856 & 0.038 & 0.825 & 0.090 & 0.603 \\
\hline BMI & 0.206 & 0.229 & 0.202 & 0.239 & 0.232 & 0.173 \\
\hline WHR & 0.272 & 0.108 & 0.272 & 0.109 & 0.277 & 0.102 \\
\hline Cholesterol & -0.190 & 0.268 & -0.106 & 0.540 & -0.108 & 0.532 \\
\hline Glycerin trilaurate & 0.363 & 0.030 & 0.333 & 0.047 & 0.362 & 0.030 \\
\hline High-density lipoprotein & -0.185 & 0.279 & -0.148 & 0.389 & -0.105 & 0.541 \\
\hline Low-density lipoprotein & -0.288 & 0.089 & -0.216 & 0.206 & -0.215 & 0.207 \\
\hline Growth hormone & 0.278 & 0.101 & 0.304 & 0.072 & 0.164 & 0.339 \\
\hline Fasting insulin & -0.148 & 0.390 & -0.187 & 0.274 & -0.116 & 0.500 \\
\hline Fasting C-peptide & 0.030 & 0.864 & 0.021 & 0.905 & -0.002 & 0.989 \\
\hline Insulin of $2 \mathrm{~h}$ after meal & -0.296 & 0.080 & -0.252 & 0.139 & -0.346 & 0.039 \\
\hline $\mathrm{C}$-peptide of $2 \mathrm{~h}$ after meal & -0.059 & 0.731 & 0.046 & 0.792 & -0.027 & 0.874 \\
\hline IGF-1 & 0.029 & 0.865 & -0.027 & 0.875 & 0.022 & 0.897 \\
\hline IGFBP-3 & -0.716 & 0.000 & -0.688 & 0.000 & -0.699 & 0.000 \\
\hline DHEA-S & 0.108 & 0.532 & 0.107 & 0.536 & 0.010 & 0.954 \\
\hline Leptin & - & - & 0.944 & 0.000 & 0.943 & 0.000 \\
\hline ADP & 0.944 & 0.000 & - & - & 0.891 & 0.000 \\
\hline Visfatin & 0.943 & 0.000 & 0.891 & 0.000 & - & - \\
\hline
\end{tabular}

BMI = body mass index; WHR = waist-to-hip ratio; IGF-1 = insulin-like growth factor-1; IGFBP-3 = insulin-like growth factor-binding protein-3; DHEA-S = dehydroepiandrosterone sulfate.

\section{Logistic regression analysis of serum leptin level}

Logistic regression analysis demonstrated that after age and gender were controlled, increased leptin $(\mathrm{OR}=1.731 ; 95 \% \mathrm{CI}=1.225-2.447 ; \mathrm{P}=0.002), \mathrm{ADP}(\mathrm{OR}=1.105 ; 95 \% \mathrm{CI}$ $=1.032-1.184 ; \mathrm{P}=0.004)$, and visfatin $(\mathrm{OR}=1.350 ; 95 \% \mathrm{CI}=1.168-1.562 ; \mathrm{P}<0.05)$ were independent risk factors for adult GHD.

\section{DISCUSSION}

This study involved adult GHD patients diagnosed by ITT and normal healthy adults. By comparing disease cases, we determined that there was a mutual relationship between altered levels of leptin, ADP, and visfatin in adult GHD patients and BMI, WHR, and blood fat; we discuss the significance of abnormal fat factors in adult GHD patients. The difference of indices between GHD patients and the controls was compared using the height, weight, WHR, blood fat level, and leptin, ADP, and visfatin levels of the two groups. The correlation between leptin, ADP, and visfatin levels and other body indices was investigated, and we discuss whether leptin, ADP, and visfatin are risk factors for GHD.

A protein product of the fat gene, the main effect of leptin is regulating fat stability. When peripheral fat is increased, the level of blood leptin is enhanced to decrease appetite, increase body fat consumption, and reduce weight as effected by multiple neuroendocrine hormones (Reinehr et al., 2009). In this study, the leptin levels in adult GHD patients were higher than those of the healthy controls. Previous reports contain inconsistencies because they were influenced by factors such as small sample size, significant discrepancies between body indices such as gender and age, and inconsistent baseline data between the GHD and control groups. The sample size in this study was larger than that of previous studies, and the baselines for gender and age were consistent, which reduced the influence of interfering factors on the results. Our results are more reliable. However, a large-scale study is required to 
verify our findings to derive a more accurate and trustworthy conclusion.

There is no difference in day- or night-time ADP secretions. ADP concentration in the blood is $5-30 \mu \mathrm{g} / \mathrm{mL}$ higher than that in plasma. As the first downregulating fat cell factor in obese patients, ADP is the only negative regulatory hormone of the fat cell factors, exerting anti-inflammatory and anti-atherosclerosis effects, reducing blood glucose and increasing insulin sensitivity. ADP reduces blood glucose in a dose-dependent manner (Yoda-Murakami et al., 2001). ADP can predict the level of HDL in type 2 diabetes mellitus independently of other common risk factors of metabolism. ADP could be a potential new target drug for curing fat metabolism disorders (Krakoff et al., 2003). This study demonstrated that ADP levels in adult GHD patients are higher than those in healthy individuals, which corresponds to the conclusion drawn by Oliveira et al. (2010). Currently, there is little research on ADP levels in adult GHD patients owing to the small number of subjects, the absence of reference hypophysis hormone levels, and different methods.

As a protein cell factor secreted by fat tissue, visfatin acts by decreasing blood glucose, as insulin does. Visfatin can induce triglycerides accumulation in preadipocytes by accelerating triglycerides synthesis through fat and glucose storage (Fukuhara et al., 2005). We found that visfatin levels in adult GHD patients were higher than those in healthy individuals.

In addition, there was a significant positive correlation among leptin, ADP, and visfatin. The positive correlation between then remained even after adjustment (or correction) of various factors by multiple logistic regression. Therefore, leptin, ADP, and visfatin are risk factors for adult GHD. Statistical analysis determined that the risk for GHD in adults with increased leptin, ADP, and visfatin levels was $1.731,1.105$, and 1.35 times, respectively, higher than that for adults with normal leptin, ADP, and visfatin levels.

\section{REFERENCES}

Alghasham AA and Barakat YA (2008). Serum visfatin and its relation to insulin resistance and inflammation in type 2 diabetic patients with and without macroangiopathy. Saudi Med. J. 29: 185-192.

Bulow B, Hagmar L, Eskilsson J and Erfurth EM (2000). Hypopituitary females have a high incidence of cardiovascular morbidity and an increased prevalence of cardiovascular risk factors. J. Clin. Endocrinol. Metab. 85: 574-584.

Fukuhara A, Matsuda M, Nishizawa M, Segawa K, et al. (2005). Visfatin: a protein secreted by visceral fat that mimics the effects of insulin. Science 307: 426-430.

Krakoff J, Funahashi T, Stehouwer CD, Schalkwijk CG, et al. (2003). Inflammatory markers, adiponectin, and risk of type 2 diabetes in the Pima Indian. Diabetes Care 26: 1745-1751.

McCallum RW, Petrie JR, Dominiczak AF and Connell JM (2002). Growth hormone deficiency and vascular risk. Clin. Endocrinol. 57: 11-24.

Oliveira CR, Salvatori R, Meneguz-Moreno RA, Aguiar-Oliveira MH, et al. (2010). Adipokine profile and urinary albumin excretion in isolated growth hormone deficiency. J. Clin. Endocrinol. Metab. 95: 693-698.

Reinehr T, Kleber M, de Sousa G and Andler W (2009). Leptin concentrations are a predictor of overweight reduction in a lifestyle intervention. Int. J. Pediatr. Obes. 4: 215-223.

Yoda-Murakami M, Taniguchi M, Takahashi K, Kawamata S, et al. (2001). Change in expression of GBP28/adiponectin in carbon tetrachloride-administrated mouse liver. Biochem. Biophys. Res. Commun. 285: 372-377. 